\title{
Solid-state light-phase detector
}

Tim Paasch-Colberg ${ }^{1}$, Agustin Schiffrin ${ }^{1, a}$, Nicholas Karpowicz ${ }^{1}$, Stanislav Kruchinin ${ }^{1}$, Özge Sağlam², Sabine Keiber ${ }^{1}$, Olga Razskazovskaya ${ }^{1}$, Sascha Mühlbrandt ${ }^{1, b}$, Ali Alnaser ${ }^{1,3,4}$, Matthias Kübel ${ }^{1}$, Vadym Apalkov ${ }^{5}$, Daniel Gerster ${ }^{2}$, Joachim Reichert ${ }^{2}$, Tibor Wittmann ${ }^{1,6}$, Johannes V. Barth ${ }^{2}$, Mark I. Stockman ${ }^{5}$, Ralph Ernstorfer ${ }^{7}$, Vladislav S. Yakovlev ${ }^{1,6}$, Reinhard Kienberger, ${ }^{1,2}$, and Ferenc Krausz ${ }^{1,6}$

${ }^{1}$ Max-Planck-Institut für Quantenoptik, Hans-Kopfermann-Str. 1, D-85748 Garching,

Germany

${ }^{2}$ Physik-Department, Technische Universität München, James-Franck-Str., D-85748

Garching, Germany

${ }^{3}$ Physics Department, POB2666 American University of Sharjah-Sharjah, United Arab

\section{Emirates}

${ }^{4}$ Faculty of Physics and Astronomy, King Saud University, Riyadh, Saudi-Arabia

${ }^{5}$ Department of Physics and Astronomy, Georgia State University, Atlanta, Georgia 30340, USA

${ }^{6}$ Fakultät für Physik, Ludwig-Maximilians-Universität, Am Coulombwall 1, D-85748

Garching, Germany

${ }^{7}$ Fritz-Haber-Institut der Max-Planck-Gesellschaft, Faradayweg 4-6, D-14195 Berlin, Germany

\footnotetext{
${ }^{a}$ Present addresses: Quantum Matter Institute, University of British Columbia, Vancouver, British Columbia, V6T 1Z4 Canada, and Department of Physics and Astronomy, University of British Columbia,Vancouver, British Columbia, V6T 1Z1 Canada

${ }^{\mathrm{b}}$ Present address: Institute of Microstructure Technology (IMT), Karlsruhe Institute of Technology (KIT), D76021 Karlsruhe, Germany
} 
Attosecond science relies on the use of intense, waveform-controlled, few-cycle laser pulses [1] to control extreme nonlinear optical processes taking place within a fraction of an optical period. A number of techniques are available for retrieving the amplitude envelope and chirp of such few-cycle laser pulses. However, their full characterization requires the detection of the absolute offset between the rapidly-oscillating carrier wave and the pulse envelope, the carrier-envelope phase (CEP). So far, this has only been feasible with photoelectron spectroscopy relying on complex vacuum setups [2-4]. Here, we present a technique that enables the detection of the CEP of few-cycle laser pulses in ambient conditions. It is based on the CEP-dependence of directly-measurable electric currents generated by the electric field of light in a metal-dielectric-metal nanojunction. The device holds promise for routine measurement and monitoring of the CEP in attosecond laboratories.

The electric field of a laser pulse can be described as $F(t)=\frac{1}{2} \tilde{F}(t) e^{-i\left(\omega_{\mathrm{L}} t+\varphi_{\mathrm{CE}}\right)}+$ c.c., where $\omega_{\mathrm{L}}$ is the carrier frequency and the complex amplitude $\tilde{F}(t)=F_{0} f(t) e^{-i \varphi(t)}$ comprises the normalized real field envelope, the peak amplitude $F_{0}$, and the time-dependent phase $\varphi(t)$. The carrier-envelope phase (CEP) $\varphi_{\mathrm{CE}}$ describes the offset between the carrier wave oscillating at $\omega_{L}$ and the maximum of the real field envelope $f(t)$. If the pulse duration, defined as the full-width at half-maximum of $f^{2}(t)$ (the square of the envelope) becomes comparable to the carrier period $2 \pi / \omega_{\mathrm{L}}$ of the waveform, $\varphi_{\mathrm{CE}}$ affects the evolution of nonlinear electron phenomena driven by $F(t)$, including high-order harmonic generation [5, 6], ionization of atoms and molecules [7-9], interaction with plasmas [10], as well as photoemission from metals [11, 12] and nanoparticles [13]. Attosecond control over these processes calls for control and full characterization of the applied electric field $F(t)$.

Attosecond streaking [14] permits complete measurement of the vector potential of a laser pulse, from which $F(t)$ is easily obtained as its temporal derivative. Alternatively, the combination of conventional pulse characterization techniques [15-17] acquiring the normalized complex amplitude $\tilde{F}(t) / F_{0}=f(t) e^{-i \varphi(t)}$ and a measurement of the CEP, $\varphi_{\mathrm{CE}}$, may provide access to the full waveform. While relative changes of the CEP can be detected by spectral characterization of mixed fields using simple and inexpensive setups [18-21], tracking down its absolute value so far is based on nonlinear photoemission from atoms or 
solids with stereo above-threshold ionization (stereo-ATI) [2-4] and attosecond streaking [14, 17], both requiring rather complex, space-consuming vacuum apparatus.

Here we report the first method permitting absolute CEP detection with a solid-state detector applicable in ambient conditions. Recently, we have shown that the strong electric field of an intense, linearly-polarized, visible/near-infrared (VIS/NIR), few-cycle laser pulse can rapidly increase the (ac) conductivity of a solid insulator, allowing electric currents to be induced and switched with the field of visible light [22]. In these experiments, we exposed amorphous silicon dioxide (bandgap $E_{\mathrm{g}} \approx 9 \mathrm{eV}$ ) to a strong, controlled electric field $F(t)$ of a few-cycle pulse with a carrier photon energy of $\hbar \omega_{\mathrm{L}} \approx 1.7 \mathrm{eV}$. The resultant transient nonlinear increase in electric polarization allows the few-cycle field to drive a current through two unbiased gold electrodes connected to the sample. Because the effect is controlled directly by $F(t)$, it is sensitive to the CEP [22].

This sensitivity can be utilized for the measurement of $\varphi_{\mathrm{CE}}$ if and only if the following two conditions are fulfilled: (i) the current vs. $\varphi_{\mathrm{CE}}$ dependence is calibrated and (ii) this calibration is robust against variations of the pulse intensity. This work addresses and fulfills these conditions via a series of careful experiments and numerical simulations. We have used sub-4-fs $\left(\sim 1.5\right.$-cycle) VIS/NIR laser pulses carried at $\lambda_{\mathrm{L}} \sim 0.75 \mu \mathrm{m}$ to study the $\varphi_{\mathrm{CE}}-$ dependence of optical-field-induced electric currents in a metal-dielectric-metal junction and compared it with measurements simultaneously performed with a stereo-ATI phasemeter [5], see Fig. 1. The junction was exposed to a strong optical field at normal incidence, with perpendicular polarization of $F(t)$, cf. Fig. 1(a)-(b). The peak electric field amplitude was set at $F_{0} \approx 1.24 \mathrm{~V} / \AA$, by focusing pulses of $\sim 4 \mu \mathrm{J}$ energy. This is only $\sim 1 \%$ of the total pulse energy of the utilized few-cycle laser system [23]. All experiments reported herein were performed in vacuum due to requirements of the stereo-ATI measurements. However, CEP detection via optical field-induced currents in a solid dielectric can be achieved in ambient conditions, as demonstrated in Ref. [22].

The stabilized CEP of the laser pulses was modulated to allow every second pulse in the train to have the same $\varphi_{\mathrm{CE}}$ (i.e. $\varphi_{\mathrm{CE}}$ was altered by $\pi$ for consecutive pulses). This specific variation of $\varphi_{\mathrm{CE}}$ is beneficial for the isolation of CEP-dependent electric currents using a lock-in amplifier for removal of CEP-independent background signals (see Methods 
Summary). In the following, " $\varphi_{\mathrm{CE}}$ " always denotes the CEP of odd-numbered pulses. However, recent experiments with an improved geometry of the solid-state device lead to a reduction of the $\varphi_{\mathrm{CE}}$-independent charge per pulse by about two orders of magnitude, enabling a direct readout of the $Q_{\mathrm{P}}\left(\varphi_{\mathrm{CE}}\right)$ without the requirement of a lock-in amplifier.

The CEP was continuously adjusted by changing the propagation length $\Delta l$ of the pulses through a pair of fused silica wedges [21]. The value of $\Delta l$ for which the CEP is changed by $\Delta \varphi_{\mathrm{CE}}=2 \pi$ is $\Delta l_{2 \pi \text { (Sellmeier) }} \cong 51 \mu \mathrm{m}$ according to the Sellmeier equation. The value $\Delta l=0 \mu \mathrm{m}$ is defined as the propagation length where the pulse duration is minimum, i.e. an increase of $|\Delta l|$ results in an increase of the pulse duration due to (small) changes in group-delay dispersion.

The CEP-dependent fraction $Q_{\mathrm{P}}$ of the total charge (i.e. the induced current integrated over an interval longer than the laser pulse) flowing through the external circuit per laser shot was measured with a lock-in detection apparatus (see Methods Summary) as a function of $\Delta \varphi_{\mathrm{CE}}$. Fig. 2(b) plots the resultant $Q_{\mathrm{P}}$ as a function of $\Delta l$. Note that the values of $Q_{\mathrm{P}}$ exceed by more than an order of magnitude those acquired in previous measurements, see Fig. 2(a) in Ref. [22], owing to improvements of the sample quality. $Q_{\mathrm{P}}(\Delta l)$ shows a clean oscillatory behavior with its maximum at $\Delta l=0 \mu \mathrm{m}$ and a period of $\Delta l_{2 \pi(\exp )} \cong 56 \mu \mathrm{m}$, which is close to $\Delta l_{2 \pi(\text { Sellmeier })}$. This discrepancy is attributed to the non-optimal alignment of the dispersive wedge with respect to the beam propagation direction. Hence, $Q_{\mathrm{P}}$ switches polarity for $\Delta \varphi_{\mathrm{CE}}=\pi$, as intuitively expected. This establishes the link between magnitude and direction of the current and the electric field $F(t)$ of the laser. The observed decay of the amplitude of $Q_{\mathrm{P}}(\Delta l)$ with the increase of $|\Delta l|$ is a consequence of dispersive pulse broadening inside the glass wedges. However, in our experiments $Q_{\mathrm{P}}(\Delta l)$ was still detectable above the noise level for values of $|\Delta l|>400 \mu \mathrm{m}$, corresponding to a pulse duration of more than 9 fs (FWHM of the time-dependent cycle-averaged intensity).

Subsequently, $Q_{\mathrm{P}}$ was calibrated with respect to the absolute CEP of the laser pulse via stereo-ATI measurements performed with identical pulses [4]. After the measurement of $Q_{\mathrm{P}}(\Delta l)$ with the solid-state device, a mirror was inserted into the beam path, deflecting the 
pulses into a stereo-ATI apparatus located - together with the solid-state detector - in the same vacuum chamber (Fig. 1). Here, the CEP of the incident laser pulse was detected by analyzing the kinetic energy distribution of electrons that are photoemitted from Xe atoms, see Methods Summary. An uncertainty due to a Gouy phase shift in both foci can be neglected since in both experiments, the sample was placed exactly in the region of the highest laser intensity.

We set 17 different propagation lengths $\Delta l$, ranging from $-21.5 \mu \mathrm{m}$ to $+27.5 \mu \mathrm{m}$. For each of them, 500 single-shot stereo-ATI measurements were performed. Because consecutive laser pulses had a CEP-shift of $\pi$, which is only required for the accurate detection of $Q_{\mathrm{P}}(\Delta l)$ , only spectra from odd-numbered pulses were considered for the stereo-ATI measurements. As shown in [4], $\varphi_{\mathrm{CE}}$ can then be reconstructed by calculating two asymmetry parameters $(X, Y)$ by integrating the averaged time-of-flight spectra $n_{\mathrm{L}, \mathrm{R}}\left(t_{\mathrm{TOF}}\right)$ of the electrons photoemitted from Xe atoms by the intense few-cycle VIS/NIR pulses in two different regions. The parametric plot of $(X, Y)$ in Fig. 2(a) was obtained by calculating, for each value of $\Delta l, \quad X=\left(P_{\mathrm{L}}^{(X)}-P_{\mathrm{R}}^{(X)}\right) /\left(P_{\mathrm{L}}^{(X)}+P_{\mathrm{R}}^{(X)}\right) \quad$ and $\quad Y=\left(P_{\mathrm{L}}^{(Y)}-P_{\mathrm{R}}^{(Y)}\right) /\left(P_{\mathrm{L}}^{(Y)}+P_{\mathrm{R}}^{(Y)}\right)$, where $P_{\mathrm{L}, \mathrm{R}}^{(X, Y)}=\int_{t_{1}^{(X, Y)}}^{t_{2}^{(X, Y)}} n_{\mathrm{L}, \mathrm{R}}\left(t_{\mathrm{TOF}}\right) d t_{\mathrm{TOF}}$. The photoelectron spectra $n_{\mathrm{L}, \mathrm{R}}\left(t_{\mathrm{TOF}}\right)$ were measured with the left (L) and right (R) micro-channel plates (MCPs) of the set-up in Fig. 1. The values for the integration were $t_{1}^{X}=27 \mathrm{~ns}, t_{2}^{X}=t_{1}^{Y}=31 \mathrm{~ns}$ and $t_{2}^{Y}=45 \mathrm{~ns}$, respectively. These intervals were chosen and optimized for high phase asymmetry.

A theoretical parametric plot $\left(X_{\mathrm{TDSE}}, Y_{\mathrm{TDSE}}\right)$ [blue in Fig. 2(a)] was calculated by numerically solving the three-dimensional time-dependent Schrödinger equation for valence electrons in isolated Xe atoms exposed to a strong few-cycle optical electric field $F(t)$ with well-defined absolute CEP (see Methods Summary for details). By associating an experimental pair $(X, Y)$ to a theoretical pair $\left(X_{\mathrm{TDSE}}, Y_{\mathrm{TDSE}}\right)$ via a radial angle $\theta=\arctan \left(Y_{\mathrm{TDSE}} / X_{\mathrm{TDSE}}\right)=\arctan (Y / X)$ in Fig. 2(a), and since each theoretical pair $\left(X_{\mathrm{TDSE}}, Y_{\mathrm{TDSE}}\right)$ corresponds to an absolute CEP, it is possible to associate a well-defined $\varphi_{\mathrm{CE}}$ to an experimental pair of parameters $(X, Y)$.

To calibrate $Q_{\mathrm{P}}(\Delta l)$ with respect to the absolute CEP of the incident laser pulses, the experimental pair $(X, Y)$ at the maximum value $Q_{\mathrm{P}}(\Delta l=0)$ of the CEP-dependent 
transferred charge was used. The upper horizontal scale in Fig. 2(b) depicts the obtained calibration. $Q_{\mathrm{P}}\left(\varphi_{\mathrm{CE}}\right)$ oscillates with a period of $2 \pi$, showing a relatively small error and high contrast with respect to the background noise and has its maximum at $\varphi_{\mathrm{CE}}^{\max }=(0.98 \pm 0.02) \pi$. An important self-consistency check can now be performed by comparing the CEP retrieved from the stereo-ATI and the - meanwhile calibrated - solid-state measurement at various values of $\Delta l$. Fig. 2(c) shows this comparison and reveals a very good agreement between the two methods. The error of the reported $\varphi_{\mathrm{CE}}$-detection method is on the same order as the error of the established stereo-ATI technique. Therefore it lends itself to the determination of the absolute $\varphi_{\mathrm{CE}}$ of single few-cycle laser pulses, as long as there is an unambiguous relationship between measured current and pulse $\varphi_{\mathrm{CE}}$.

We have compared the results of the solid-state-based phase retrieval with the predictions of two quantum mechanical models. The first model, which was earlier employed in Ref. [24] to describe the ultrafast increase in conductivity of $\mathrm{SiO}_{2}$ nanojunctions, is based on the nearestneighbor tight-binding approximation. The second model, presented in detail in Ref. [25], describes quantum dynamics in a one-dimensional pseudopotential (see the Methods Summary for details). In both models, the electric field in the medium is evaluated selfconsistently with the induced polarization. As revealed in Fig. 2(b), an excellent agreement is achieved between the measured data and the predictions of both theoretical models.

Reliable determination of $\varphi_{\mathrm{CE}}$ at different laser intensities via the detection of $Q_{\mathrm{P}}$ calls for low sensitivity of the measurement result with respect to intensity variations. In order to assess this sensitivity, we have performed a series of measurements of $Q_{\mathrm{P}}$ as a function of $\varphi_{\mathrm{CE}}$ [as in Fig. 2(b)] for different laser field strengths $F_{0}$. With varying $F_{0}$, we observe a displacement of the $Q_{\mathrm{P}}$-VS- $\varphi_{\mathrm{CE}}$ curve with respect to $\varphi_{\mathrm{CE}}$, i.e., when $F_{0}$ varies, the value of $\varphi_{\mathrm{CE}}$ for which $Q_{\mathrm{P}}$ is maximum changes. Figure 2(d) shows this shift in the CEP scale with respect to the calibrated scale obtained at $F_{0} \approx 1.24 \mathrm{~V} / \AA$, along with the prediction of the theoretical models. For field strengths in excess of $1 \mathrm{~V} / \mathrm{A}$, good agreement is found between theory and experiment.

Both theory and experiment reveal significant shifts below $1 \mathrm{~V} / \AA ̊ \AA$, rendering this intensity regime unsuitable for CEP measurements by the solid-state detector. In stark contrast, for 
field strengths above $1 \mathrm{~V} / \AA$, shifts of the $Q_{\mathrm{P}}$-vs- $\varphi_{\mathrm{CE}}$ curves with varying $F_{0}$ remain small [Fig. 2(b)]. Only the amplitude of $Q_{\mathrm{P}}\left(\varphi_{\mathrm{CE}}\right)$ changes significantly and nonlinearly (cf. Fig. 2(b) in Ref. [22]). The phase calibration is robust with an accuracy on the order of $\pm 310 \mathrm{mrad}$ in a range from $F_{0} \approx 1 \mathrm{~V} / \AA$ to $2.5 \mathrm{~V} / \AA$, corresponding to $\mathrm{I}_{0} \approx(1-7) \times 10^{13} \mathrm{~W} / \mathrm{cm}^{2}$ (see upper axis in Fig. 2(d) for an intensity scale), or in other words, to $\pm 75 \%$ around $4 \times 10^{13} \mathrm{~W} / \mathrm{cm}^{2}$. This is a remarkable result since conventional techniques for CEP detection and stabilization show a much bigger sensitivity to fluctuations of the pulse energy [26, 27], where an energy fluctuation of $1 \%$ induced a $\varphi_{\mathrm{CE}}$-shift of $160 \mathrm{mrad}$.

However, the measurement of $Q_{\mathrm{P}}\left(\varphi_{\mathrm{CE}}\right)$ from a single metal-dielectric-metal junction as described above allows for determining the absolute $\varphi_{\mathrm{CE}}$ of a single laser pulse with a remaining phase ambiguity only, cf. Fig. 2(b): As a matter of fact, within the $\varphi_{\mathrm{CE}}$-interval of $0-2 \pi$, two different values of $\varphi_{\mathrm{CE}}$ can give the same $Q_{\mathrm{P}}\left(\varphi_{\mathrm{CE}}\right)$, e.g., $Q_{\mathrm{P}}(0.7 \pi)=Q_{\mathrm{P}}(1.2 \pi)$. For stabilized $\varphi_{\mathrm{CE}}$, this can be resolved by subsequent measurements of $Q_{\mathrm{P}}\left(\varphi_{\mathrm{CE}}\right)$ with slightly changed values of $\varphi_{\mathrm{CE}}$ around its nominal value, cf. Fig. 3(b). In the absence of CEPstabilization, this ambiguity must be removed within one laser shot. This could be resolved by the use of two identical junctions, exposed simultaneously to pulses of equal field strength from the same source, whose relative CEPs differ by $\pi / 2$ [Fig. 3(c)]. Analogously to the photoelectron signals of the two independent MCPs in the stereo-ATI setup, the pair of measured electronic signals $Q_{\mathrm{P}}^{(1)}\left(\varphi_{\mathrm{CE}}\right)$ and $Q_{\mathrm{P}}^{(2)}\left(\varphi_{\mathrm{CE}}\right)$ from both junctions is unambiguously related to the absolute CEP of the initial laser pulse, $\varphi_{\mathrm{CE}}$. For example in Fig. 3(a), $Q_{\mathrm{P}}^{(1)}(0.7 \pi)=Q_{\mathrm{P}}^{(1)}(1.2 \pi)$, but $Q_{\mathrm{P}}^{(2)}(0.7 \pi) \neq Q_{\mathrm{P}}^{(2)}(1.2 \pi)$, allowing for disambiguation and singleshot CEP detection via $\varphi_{C E} \cong-\arg \left(Q_{\mathrm{P}}^{(1)}+i Q_{\mathrm{P}}^{(2)}\right)$. This method of using the signals from both junctions also minimizes any potential phase errors arising from amplitude changes between consecutive laser pulses.

In summary, we have demonstrated the feasibility of measuring the absolute CEP of fewcycle laser fields with a solid-state device. Its ability to function in ambient conditions at relatively low laser energy, its stability against fluctuations of the laser energy and also providing a direct electronic readout makes this approach uniquely powerful. Furthermore, the 
lifetime of such a device is not limited by laser induced damage. Thus, this light phase detector holds promise as a valuable addition to the toolbox of attosecond metrology and spectroscopy, not only for absolute CEP detection, but also for CEP stabilization.

\section{Methods Summary}

Source of ultrashort laser pulses. Linearly polarized laser pulses in the visible/near-infrared with $\sim 400 \mu \mathrm{J}$ pulse energy and controllable CEP are generated at a $3 \mathrm{kHz}$ repetition rate with a customized titanium-sapphire chirped-pulse amplifier (CPA) system [23]. The ultra-broadband spectrum of the laser pulses ranges from 500-1050 nm, yielding a sub-4 fs pulse duration (full-width at half-maximum of the temporal intensity profile). This corresponds to a $\sim 1.5$-cycle pulse at a carrier wavelength of $\sim 0.75 \mu \mathrm{m}$. The laser pulses can either be focused onto the solid-state system or into the gas cell of the stereo-ATI device, reaching a cycle averaged peak intensity of up to $1 \times 10^{14} \mathrm{~W} / \mathrm{cm}^{2}$.

Photoactive metal-dielectric circuit. The solid-state device on which the experiments on ultrafast current in the solid-state have been performed consists of a nanoscaled metal-dielectric-metal junction. Such structures are fabricated by cleaving amorphous $\mathrm{SiO}_{2}($ Crystec $\mathrm{GmbH})$ and coating the adjacent surfaces of the atomically-sharp cleaved silica edge with $\sim 50 \mathrm{~nm}$ of gold evaporated at grazing incidence. With this procedure, a pair of regular straight gold electrodes isolated from each other by a $\mathrm{SiO}_{2}$ nanotrench with a width on the order of $\sim 50 \mathrm{~nm}$ is obtained. This junction is coated with an additional sputtered $\mathrm{SiO}_{2}$ nanofilm of $\sim 200 \mathrm{~nm}$ to embed the gold electrodes in a homogeneous silica matrix. During the experiment no bias voltage was applied to the electrodes.

Detection scheme for optically induced CEP-dependent electrical signals. The experimental data on optically induced ultrafast currents inside a solid-state device presented in this work are the CEPdependent component of the optically generated electric signal in the nanojunction. This CEPdependent component is filtered from CEP-independent contributions of the electric signal (e.g. electric currents caused across the junction by CEP-independent photoionization of gold due to imbalanced irradiation of the pair of electrodes) that are up to two orders of magnitude bigger by applying a lock-in technique. For this purpose the pulse train of the CPA system is synchronized such that two consecutive laser pulses have a CEP-change of $\pi$, resulting in a modulation of the CEP at half the repetition rate of the laser pulse train. The optically induced electric signal in the solid-state device is amplified using a high-gain I/V-converter with a bandwidth that supports the laser pulse train. With a lock-in amplifier referenced to the modulation frequency of the CEP-modulation, the CEP-dependent component $Q_{\mathrm{P}}$ of the electric signal is extracted. The signal-to-noise ratio of the lockin detection of $Q_{\mathrm{P}}$ is about 350 . 
Stereo-ATI device for detecting the absolute CEP. The laser pulses are focused into a gas cell filled with xenon at a pressure of $\sim 3.5 \times 10^{-3}$ mbar, where the strong laser field leads to above-thresholdionization of the Xe atoms and the subsequent acceleration of the released electrons in the laser field. A CEP-dependent signature is imprinted upon the kinetic energy distribution of the electrons accelerated in opposite direction along the laser polarization. Those energy distributions are recorded by detecting the electrons' flight time through a $155 \mathrm{~mm}$ long vacuum tube (pressure: $\sim 2 \times 10^{-6} \mathrm{mbar}$ ) on both sides of the gas cell by using a micro-channel plate at the end of each vacuum tube and a photodiode behind the gas cell to detect the time of the laser pulse incidence. Thus, the absolute CEP of the incoming laser pulses can be reconstructed once the absolute dependence of the photoelectron spectrum on CEP is determined. This was done by numerically solving the time-dependent Schrödinger equation in the single active electron approximation $[28,29]$ using a model atomic potential known to accurately reproduce the energy structure and re-scattering behavior in strong-field photoionization [30], and averaging the resulting photoelectron spectra over intensity. This map of photoelectron energy vs. CEP was then related to the experimental data as described in the main text.

Details of the quantum-mechanical simulation. We have employed two models. The first one is the same as that described in Ref. [24]. In the second model, described in Ref. [25], we use an effective one-dimensional periodic potential

$$
U(z)=\sum_{n=-\infty}^{+\infty} c_{1}\left\{\tanh ^{2}\left[c_{2}(z+n a)\right]-1\right\}
$$

with parameters that approximate the properties of $\alpha$-quartz along the [0001] direction: the lattice constant $a=5.4 \AA, c_{1}=2.016$ at. u. , and $c_{2}=0.822$ at. u. The solution of the stationary problem yields a bandgap of $E_{\mathrm{g}} \approx 9 \mathrm{eV}$ and an effective electron mass in the lowest conduction band of $m_{\mathrm{c}} \approx 0.4 m_{0}$. The parameters $c_{1}$ and $c_{2}$ were adjusted to obtain the best agreement between simulations and experimental data. Evaluating the transferred charge $Q_{\mathrm{P}}$, we averaged over the intensity distribution in the laser spot: $Q_{\mathrm{P}} \propto \int_{0}^{\infty} q_{\mathrm{P}}\left[F_{0}(x)\right] \mathrm{d} x$, where $q_{\mathrm{P}}\left[F_{0}\right]$ is the charge density for a given peak amplitude, and we assumed a Gaussian beam profile with a center at $x=0$ and a beam waist of $50 \mu \mathrm{m}$. Our simulations indicate that the observed relative phase shift in the dependence of $Q_{\mathrm{P}}\left(\varphi_{\mathrm{CE}}\right)$ on the laser intensity [see Fig. 2(d)] is related to the interplay between two competing forces acting on electrons: the field of the laser pulse and the screening field induced by the macroscopic polarization of the dielectric. 


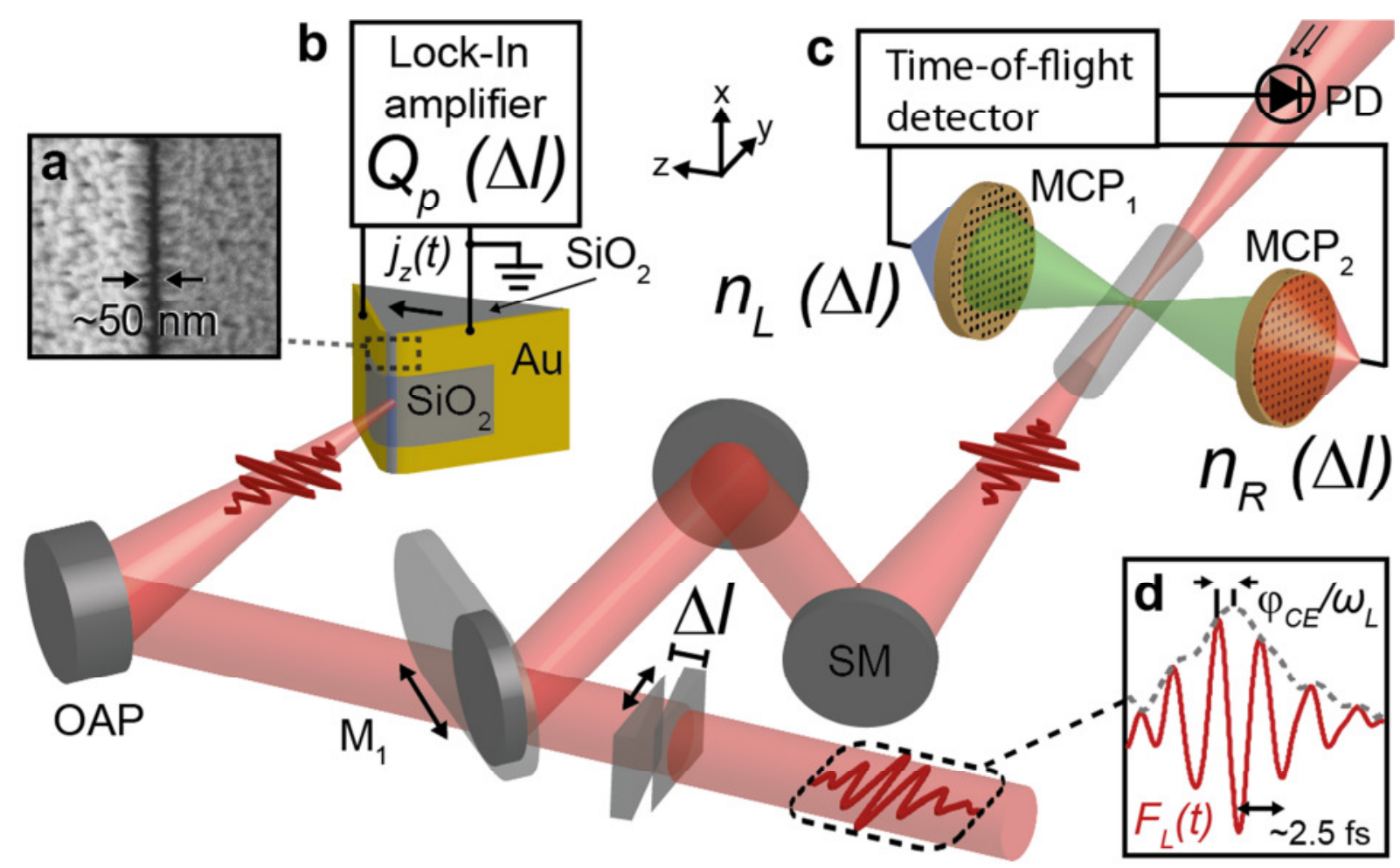

Fig. 1: Measurement of the absolute carrier-envelope phase of light pulses by optical-fieldinduced electric currents in a solid-state device. A metal-dielectric-metal junction (see (a) for a scanning electron microscope image of an exemplary junction) in vacuum is exposed to a few-cycle VIS/NIR laser pulse, focused with an off-axis parabolic mirror (OAP), with its polarization oriented perpendicular to the junction (i.e., in $z$ direction). The CEP is varied by tuning the propagation length $\Delta l$ of the pulses inside a pair of fused silica wedges. The light-field $F_{L}(t)$ mediates an increased conductivity of the dielectric, polarizing the junction and creating a current density $j_{z}(t)$ between the electrodes. $F_{L}(t)$ was determined in a previous experiment [14], cf. (d). (b) The CEP-dependent fraction $Q_{\mathrm{P}}$ of the total charge is detected with a lock-in amplifier. $Q_{\mathrm{P}}$ is calibrated with respect to the absolute CEP of the laser pulse via parallel stereo-ATI measurements (c). Here, the laser field is focused with a spherical mirror (SM) into a gas-filled cell where it ionizes Xe atoms and accelerates the photoelectrons towards a pair of micro-channel plates (MCP). The CEP-dependent time-of-flight distributions $n_{\mathrm{L}, \mathrm{R}}\left(t_{\mathrm{TOF}}, \Delta l\right)$ of the photoelectrons are determined using a photodiode (PD) for zero-time reference in left (L) and right (R) direction for various values of $\Delta l$. 


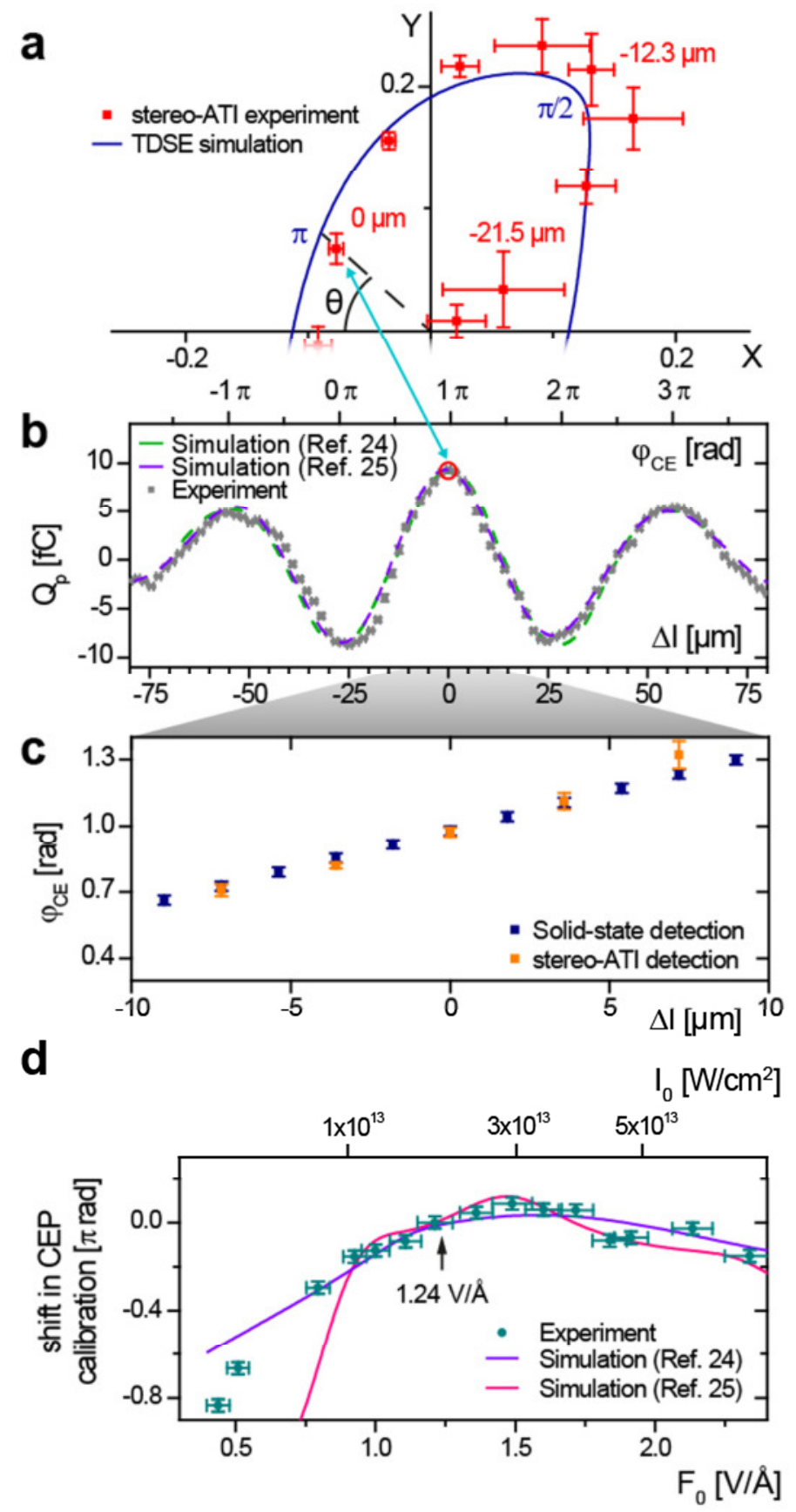

Fig. 2: Calibration of the CEP-dependent current. (a) Parametric plot of the asymmetry parameters $X$ and $Y$ of the stereo-ATI measurements (red). The error bars indicate the standard deviation of both measurements. The results of the simulation based on solving the time-dependent Schrödinger equation (TDSE) are shown in blue. The radial angle $\theta$ is used to calibrate the experimental stereoATI data. (b) $Q_{\mathrm{P}}$ (gray) shown as a function of the propagation length $\Delta l$ of the laser pulse in the dispersive fused silica wedge (lower horizontal axis). The data points represent an average of 1500 consecutive measurements at a given $\Delta l$ and $F_{0}=1.24 \mathrm{~V} / \AA$. The error bars indicate the standard deviation. Using stereo-ATI measurements from (a) at $\Delta l=0 \mu \mathrm{m}, Q_{\mathrm{P}}$ is calibrated with respect to the absolute CEP ( $\varphi_{\mathrm{CE}}$, upper horizontal axis). The results of the quantum mechanical simulations (Refs. 
[24] and [25]) are shown in green and purple. (c) The method to detect $\varphi_{\mathrm{CE}}$ with a solid-state device (blue) is compared with the detection using a stereo-ATI apparatus (orange) at different values of $\Delta l$. (d) Relative shift of $Q_{\mathrm{P}}\left(\varphi_{\mathrm{CE}}\right)$ for different values of laser field amplitude $F_{0}$ (lower axis) and of cycleaveraged peak intensity $I_{0}$ (upper), compared with the simulation results.
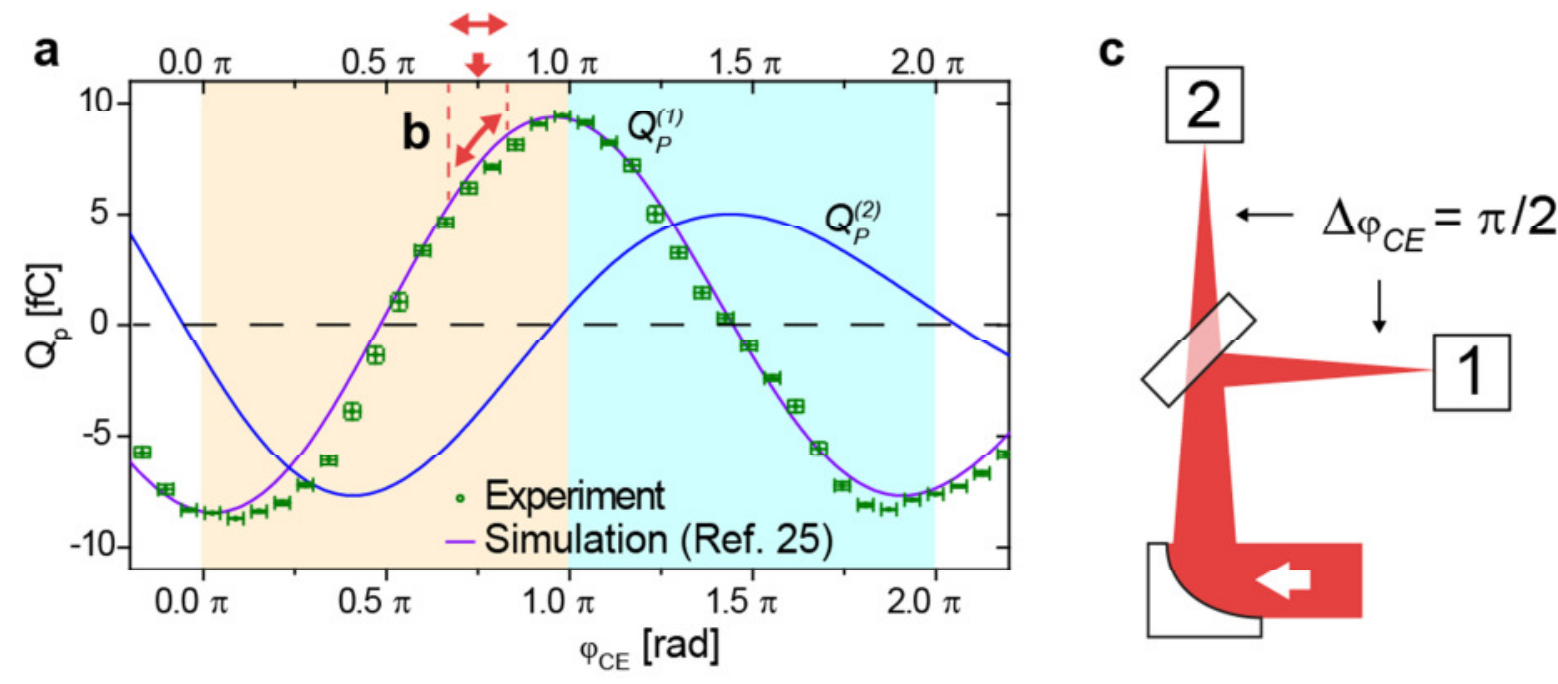

Fig. 3: Direct determination of the absolute CEP via directly measurable photocurrents. (a) The CEP-dependent component $Q_{\mathrm{P}}$ as a function of the absolute $\varphi_{\mathrm{CE}}$ of the incident laser pulse in the interval of $0-2 \pi \mathrm{rad}$. (b) The $\varphi_{\mathrm{CE}}$ of repetitive identical pulses can be detected with a single junction by slight variations of $\varphi_{\mathrm{CE}}$ around its nominal value (here, $\varphi_{\mathrm{CE}}=0.75 \pi$ is taken as an example). (c) For the $\varphi_{\mathrm{CE}}$-detection of single laser pulses with very high signal-to-noise ratio, a device consisting of two $\mathrm{Au}-\mathrm{SiO}_{2}-\mathrm{Au}$-junctions can be used. The pulse irradiating the second junction has a CEP-shift of $\pi / 2$ after transmission through a dispersive beam splitter, leading to an identical shift of the current from this junction (the corresponding simulation result is shown in blue). The sign (positive or negative) of the collected charge from the second junction $Q_{\mathrm{P}}^{(2)}$ can be used to determine if $\varphi_{\mathrm{CE}}$ is in the interval $0-\pi$ or $\pi-2 \pi$, whereas the measured charge value from the first junction $Q_{\mathrm{P}}^{(1)}$ can then be used to read out the actual value of $\varphi_{\mathrm{CE}}$. Thus, the real-time electric field of the incident laser pulse can be reconstructed. 


\section{References}

1. Krausz, F. \& Ivanov, M. Attosecond physics. Rev. Mod. Phys. 91, 163-234 (2009).

2. Paulus, G. G. et al. Absolute-phase phenomena in photoionization with few-cycle laser pulses. Nature 414, 182-184 (2001).

3. Paulus, G. G. et al. Measurement of the phase of few-cycle laser pulses. Phys. Rev. Lett. 91, 253004 (2003).

4. Wittmann, T. et al. Single-shot carrier-envelope phase measurement of few-cycle laser pulses. Nat. Phys. 5, 357-362 (2009).

5. Chipperfield, L. E., Robinson, J. S., Knight, P. L., Marangos, J. P. \& Tisch, J. W. G. The generation and utilization of half-cycle cut-offs in high harmonic spectra. Laser Photon. Rev. 4, 697-719 (2010).

6. Goulielmakis, E. et al. Single-cycle nonlinear optics. Science 320, 1614-1617 (2008).

7. Bergues, B. et al. Attosecond tracing of correlated electron-emission in non-sequential double ionization. Nat. Commun. 3, 813 (2012).

8. Dietrich, P., Krausz, F. \& Corkum, P. B. Determining the absolute carrier phase of a few-cycle laser pulse. Opt. Lett. 25, 16-18 (2000).

9. Kling, M. F. et al. Control of electron localization in molecular dissociation. Science 312, 246-248 (2006).

10. Borot, A. et al. Attosecond control of collective electron motion in plasmas. Nat. Phys. 8, 416-421 (2012).

11. Apolonski, A. et al. Observation of light-phase-sensitive photoemission from a metal. Phys. Rev. Lett. 92, 073902 (2004).

12. Kruger, M., Schenk, M. \& Hommelhoff, P. Attosecond control of electrons emitted from a nanoscale metal tip. Nature 475, 78-81 (2011).

13. Zherebtsov, S. et al. Controlled near-field enhanced electron acceleration from dielectric nanospheres with intense few-cycle laser fields. Nat. Phys. 7, 656-662 (2011).

14. Kienberger, R. et al. Atomic transient recorder. Nature 427, 817-821 (2004).

15. Trebino, R. et al. Measuring ultrashort laser pulses in the time-frequency domain using frequencyresolved optical gating. Rev. Sci. Instrum. 68, 3277-3295 (1997).

16. Iaconis, C. \& Walmsley, I. A. Spectral phase interferometry for direct electric-field reconstruction of ultrashort optical pulses. Opt. Lett. 23, $792-794$ (1998).

17. Witting, T. et al. Sub-4-fs laser pulse characterization by spatially resolved spectral shearing interferometry and attosecond streaking. J. Phys. B: At. Mol. Opt. Phys. 45, 074014 (2012).

18. Holzwarth, R. et al. Optical frequency synthesizer for precision spectroscopy. Phys. Rev. Lett. 85, 2264-2267 (2000). 
19. Reichert, J., Holzwarth, R., Udem, T. \& Hänsch, T. W. Measuring the frequency of light with mode-locked lasers. Opt. Comm. 172, 59-68 (1999).

20. Telle, H. R. et al. Carrier-envelope offset phase control: A novel concept for absolute optical frequency measurement and ultrashort pulse generation. Appl. Phys. B 69, 327-332 (1999).

21. Xu, L. et al. Route to phase control of ultrashort light pulses. Opt. Lett. 21, 2008-2010 (1996).

22. Schiffrin, A. et al. Optical-field-induced current in dielectrics. Nature 493, $70-74$ (2013).

23. Cavalieri, A. L. et al. Intense 1.5-cycle near infrared laser waveforms and their use for the generation of ultra-broadband soft-x-ray harmonic continua. New J. Phys. 9, 242 (2007).

24. Apalkov, V. \& Stockman, M. I. Theory of dielectric nanofilms in strong ultrafast optical fields. Phys. Rev. B 86, 165118 (2012).

25. Kruchinin, S. Y., Korbman, M. \& Yakovlev, V. S. Theory of strong-field injection and control of photocurrent in dielectrics and wide band gap semiconductors. Phys. Rev. B 87, 115201 (2013).

26. Kübel, M. et al. Carrier-envelope-phase tagging in measurements with long acquisition times. New J. Phys. 14, 093027 (2012).

27. Li, C. et al. Determining the phase-energy coupling coefficient in carrier-envelope phase measurements. Opt. Lett. 32, 796-798 (2007).

28. Karpowicz, N. \& Zhang, X.-C. Coherent terahertz echo of tunnel ionization in gases. Phys. Rev. Lett. 102, 093001 (2009).

29. Muller, H. G. An efficient propagation scheme for the time-dependent Schrödinger equation in the velocity gauge. Laser Phys. 9, 138-148 (1999).

30. Bian, X. B. et al. Subcycle interference dynamics of time-resolved photoelectron holography with midinfrared laser pulses. Phys. Rev. A 84, 043420 (2011).

\section{Acknowledgements}

We thank Y. Deng for technical support and fruitful discussions as well as the Munich-Centre for Advanced Photonics for financial support. A.S. acknowledges the Alexander von Humboldt Foundation and the Swiss National Science Foundation. N.K. acknowledges the Alexander von Humboldt Foundation. Ö.S. acknowledges a Marie Curie International Incoming Fellowship (Project NANOULOP, No. 302157). R.K. acknowledges an ERC Starting Grant. 


\section{Contributions}

T.P.-C., A.S., R.K., R.E. and F.K. conceived and supervised the experiments. A.S., T.P.-C., D.G., O.R. S.M., J.R., and J.V.B. participated in the sample design and fabrication. T.P.-C., A.S., N.K., A.A., Sa.K. and Ö.S. performed the measurements. St.K., V.A., M.I.S. and V.S.Y. provided theoretical description and numerical modeling of the solid-state device for phase detection. T.P.-C. and N.K. performed numerical simulations and analysis of the stereo-ATI measurements. T.W. provided the stereo-ATI phasemeter. T.P.-C., A.S., N.K., St.K., M.K., T.W., V.S.Y., R.K., R.E., and F.K. analyzed and interpreted the experimental data. All authors discussed the results and contributed to the final manuscript.

\section{Corresponding authors}

Correspondence to Tim Paasch-Colberg or Ferenc Krausz

\section{Competing financial interests}

The authors declare no competing financial interests. 